\title{
Cocaine's Appetite for Fat and the Consequences on Body Weight
}

Billing L. ${ }^{1}$ and Ersche K.D. ${ }^{1,2 凶}$

${ }^{1}$ University of Cambridge, Departments of Psychology and Psychiatry, Cambridge, U.K.

${ }^{2}$ Behavioural and Clinical Neuroscience Institute, University of Cambridge, Cambridge, U.K.

$\triangle$ Correspondence:

Dr Karen Ersche, University of Cambridge, Department of Psychiatry, Herchel Smith Building for Brain \& Mind Sciences, Cambridge Biomedical Campus, Cambridge CB2 0SZ, UK. Phone: +44 (0)1223 336587, Fax: +44 (0)1223 336581, e-mail: ke220@,cam.ac.uk

Word count: 1,909 main body and legend (excluding references), 178 abstract

Reference count: 21

Number of Figures: 1 


\begin{abstract}
For many individuals in treatment for cocaine dependence, weight gain is a substantial problem during recovery. This weight gain causes significant distress and seems to increase the risk of relapse. The mechanisms underlying cocaine's effects on weight remain elusive. It is widely assumed that this weight gain reflects a metabolic or behavioural compensatory response to the cessation of cocaine use. Here we challenge this assumption and outline potential mechanisms by which chronic cocaine use produces disturbances in the regulation of fat intake and storage, through its effects on the central and peripheral nervous systems, specifically the sympathetic nervous system. We hypothesise that the cocaine-induced alteration in fat regulation results in cocaine users developing a pronounced appetite for fatty food but keeps their fat mass low. This altered fat appetite subsequently leads to excessive weight gain when individuals enter treatment and stop using cocaine. Our aim is to shed light on the neurobiological mechanisms that may underlie the alterations in eating and fat regulation in cocaine-dependent individuals, to open up potential new avenues to support these individuals in recovery.
\end{abstract}


Whilst cocaine is best known for its reinforcing effects and addictive potential, its influence on appetite and body weight is less well understood. This is surprising, given that weight gain is a substantial problem during recovery from cocaine dependence and is associated with significant distress and poorer compliance with treatment. There are currently no guidelines for health care professionals on how to address weight change in cocaine-dependent individuals receiving treatment. Instead, clinical practice seems to be dominated by the view that this weight gain during recovery reflects a compensatory response to restore weight that was previously lost due to the appetite suppressant effects of cocaine (1). Another prevailing view is that individuals who give up drug-taking replace the drug with food, further increasing the risk of weight gain. Neither of these beliefs are really supported by scientific evidence. In fact, the pre-clinical evidence shows that cocaine's anorexigenic effects are acute and transient (2) and are shortly followed by a compensatory increase in the consumption of fat and carbohydrates (3). These pre-clinical observations are in keeping with the eating patterns that have been reported in cocaine-dependent humans $(4 ; 5)$, who show a preference for fat but without concomitant weight gain. The mechanisms underlying these effects of cocaine on eating behavior and weight remain elusive. Here we aim to turn the spotlight on the effects that cocaine exerts on the central and the peripheral, sympathetic nervous systems. These may not only elucidate a variety of metabolic disturbances possibly underpinning cocaine users' preferences for fatty food in spite of low body weight, but may also explain the excessive weight gain in abstinent cocainedependent individuals. 


\section{Central nervous effects of cocaine on fat regulation}

\subsection{Cocaine's modulatory effects on fat intake}

Cocaine is a central nervous system stimulant that blocks the reuptake of monoamines. However, its addictive potential has mainly been attributed to its ability to inhibit the dopamine transporter (DAT), thereby increasing extracellular dopamine (DA) availability in the synaptic cleft and stimulating postsynaptic dopamine receptors. It is this sharp increase in extracellular dopamine that has been linked to its euphorigenic effect and the desire to take the drug again. But could cocaine's central dopaminergic effects also influence an individual's food preference? Pre-clinical work by Thanos et al. (6) supports this hypothesis. They demonstrated that the dopamine D2 receptor (D2-R) agonist bromocriptine, which increases dopaminergic neurotransmission in the midbrain, acutely suppresses overall food intake in rats. Once these acute effects fade and the animals commence eating, they express a preference for high fat food pellets over regular food, possibly reflecting an enhanced motivational drive for fat-rich food (6). Similarities between the effects of bromocriptine and cocaine on midbrain dopamine transmission have fuelled speculations about whether the compulsive use of food and drugs may have a common biochemical origin (7).

Cocaine also inhibits the re-uptake of serotonin (5-HT) via presynaptic serotonin transporters (SERT), increasing serotonin availability in the synaptic cleft (8). Serotonergic neurons in the brainstem are known to project to hypothalamic regions including the arcuate nucleus, increasing appetite by activating postsynaptic 5-HT receptors (e.g. Htrla) of arcuate nucleus neurons (Yadav et al., 2009). Additionally, a number of knockout studies in mice found that the inhibition of these 5-HT producing brainstem neurons induced leptin-dependent anorexic effects (9). Chronic cocaine exposure may therefore enhance food consumption by modulating 
5-HT neurotransmission in the arcuate nucleus both directly, via the inhibition of SERT, and indirectly by reducing leptin production through its peripheral effects (see section 2).

The specific appetite for fat may however be modulated by cocaine's excitatory effects on the hypothalamic-pituitary-adrenal axis (HPA) through the release of hypothalamic corticotropinreleasing factor (CRF). Raised levels of circulating glucocorticoids have been linked to both an increase in the consumption of calorific food and impaired memory of the calorific ingestion, resulting in an overall increase in food intake (10). A more nuanced view on glucocorticoid hormones' influences on diet, however, differentiates the involvement of homeostatic factors such as hormones and neuroendocrine signals (i.e. insulin and neuropeptide Y) and nonhomeostatic factors such as changes in motivation on both drug-taking behaviors and eating $(11 ; 12)$. Glucocorticoids have been shown to promote the release of neuropeptide Y (NPY) whilst inhibiting the anorexigenic modulatory effects of factors like leptin (13), possibly driving the increase in appetite. Glucocorticoids also enhance insulin release and higher circulating insulin levels are associated with fatty food consumption (14). There is pre-clinical evidence suggesting that insulin may enhance the behavioral effects of cocaine (15). It is conceivable that a glucocorticoid-stimulated increase in insulin levels may also underlie cocaine users' preference for a fatty diet. Moreover, the fat-induced increase in insulin levels in cocaine users might further be reinforced by insulin's modulatory effects on dopaminergic neurotransmission (16), thereby facilitating the overeating of foods that are specifically high in fat.

Satiety signals, modulated by leptin and insulin, typically regulate the intake of fatty foods. Both of these hormones inhibit the activities of appetite-promoting orexin and NPY/AgRP neurons in the arcuate nucleus, whilst also promoting the activity of appetite-inhibiting POMC neurons. Not only do these two satiety factors converge on the same molecular signaling 
pathways, including the phosphoinositide 3-kinase (PI-3K) pathway, there also seems to be cross-talk between the biochemical responses to leptin and insulin in neurons of the arcuate nucleus (17). Thus, chronic cocaine use is likely to interfere with these two satiety signals, further facilitating an increase in the consumption of fatty foods.

\subsection{Cocaine's modulatory effects on fat storage}

Despite their high intake of foods rich in fat, cocaine-dependent individuals do not exhibit a concomitant increase in body weight. This somewhat paradoxical effect may be because of a variety of cocaine-induced metabolic disturbances that could interfere with the regulation of fat intake and storage. As outlined in Figure 1, cocaine promotes the production and the release of the neuropeptide cocaine and amphetamine regulated transcript (CART) (18), which is implicated in the regulation of feeding, satiety and body weight (19). Pre-clinical studies have shown that CART, when centrally administered, reduces food intake in the short-term, whilst changing lipid metabolism in the long-term (20). This CART-induced shift in the management of circulating lipids from deposition to mobilization and oxidation is likely to decrease the storage of body fat (20). The direct effects of cocaine on CART may thus explain cocaine users' relatively low body fat in spite of their high fat intake.

\section{The effects of cocaine through the periphery}

One of the most notable effects of cocaine use is the concomitant increase in heart rate and blood pressure, caused by increased sympathetic nerve activity through the inhibition of the noradrenaline transporter (NAT). As a potent sympathomimetic, cocaine increases the mobilization of stored lipids, metabolic rate and thermogenesis, subsequently reducing adiposity. Importantly, up-regulation of sympathetic nervous system activity has also been 
linked to a reduction in the production of leptin and increased appetite(21). Cocaine's sympathomimetic effects may therefore explain prior observations in cocaine-dependent men with low plasma leptin levels, who reported uncontrolled intake of food high in fat and carbohydrates (4). Furthermore, the appetite enhancing effects of reduced leptin production coupled with the long-term metabolic effects of cocaine-induced up-regulation of CART represent a disconnection between leptin and CART signaling in the negative feedback cycle controlling body fat accumulation (20), which might result in a reduction in body weight despite increased food consumption in cocaine-dependent individuals.

\section{Possible Clinical Implications}

This article aims to challenge prevailing beliefs regarding weight gain in recovering cocainedependent individuals. Specifically, it challenges the widely-held ideas that weight gain represents a compensatory metabolic response to a previous appetite-suppressant effect and/or, a compensatory behavioral response to the loss of a substance that was compulsively consumed. Here, we outline hypotheses about how chronic cocaine use may alter the regulation of fat intake and storage. In our view, overeating in chronic cocaine users does not start during recovery as widely assumed, but may actually develop during active cocaine use, with the drug specifically affecting the choice for the consumption of highly calorific fatty foods. This may however, go unnoticed because the consumption of fatty food does not coincide with the acute pharmacological actions of the drug, but occurs after a few hours delay, and more importantly, it is not accompanied by weight gain. Excessive weight gain only occurs upon the discontinuation of cocaine use, as the dietary preference for fat-rich food persists but the effects on fat mobilization and oxidation wane. The sudden and excessive weight gain may then come 
as a surprise to cocaine-dependent individuals, as they may not have noticed any change in their dietary food intake to which they could attribute the increase in body weight.

We believe an important clinical implication of the above is the need to incorporate these phenomena into our clinical conceptualization of cocaine dependence, and to anticipate them during abstinence and recovery. A better understanding of the exact molecular mechanisms underlying these metabolic disturbances would be vital to the development of pharmacological strategies to address these changes in eating behavior and fat metabolism in these individuals. However it is most important for both cocaine dependent individuals and the clinicians who treat them, to be aware of these metabolic effects of cocaine or enable them to address these early during the recovery process.

\section{Acknowledgements}

LB is the recipient of a Wellcome Trust Vacation Scholarship and KDE is supported by the Medical Research Council (G1000018). The authors thank Hisham Ziauddeen for his comments on the manuscript and the Medical Research Council and the Wellcome Trust for their joint support of the Behavioural and Clinical Neuroscience Institute, which provided the infrastructure for this work.

\section{Declaration of Interest}

The authors declare to have no conflicts of interest. 


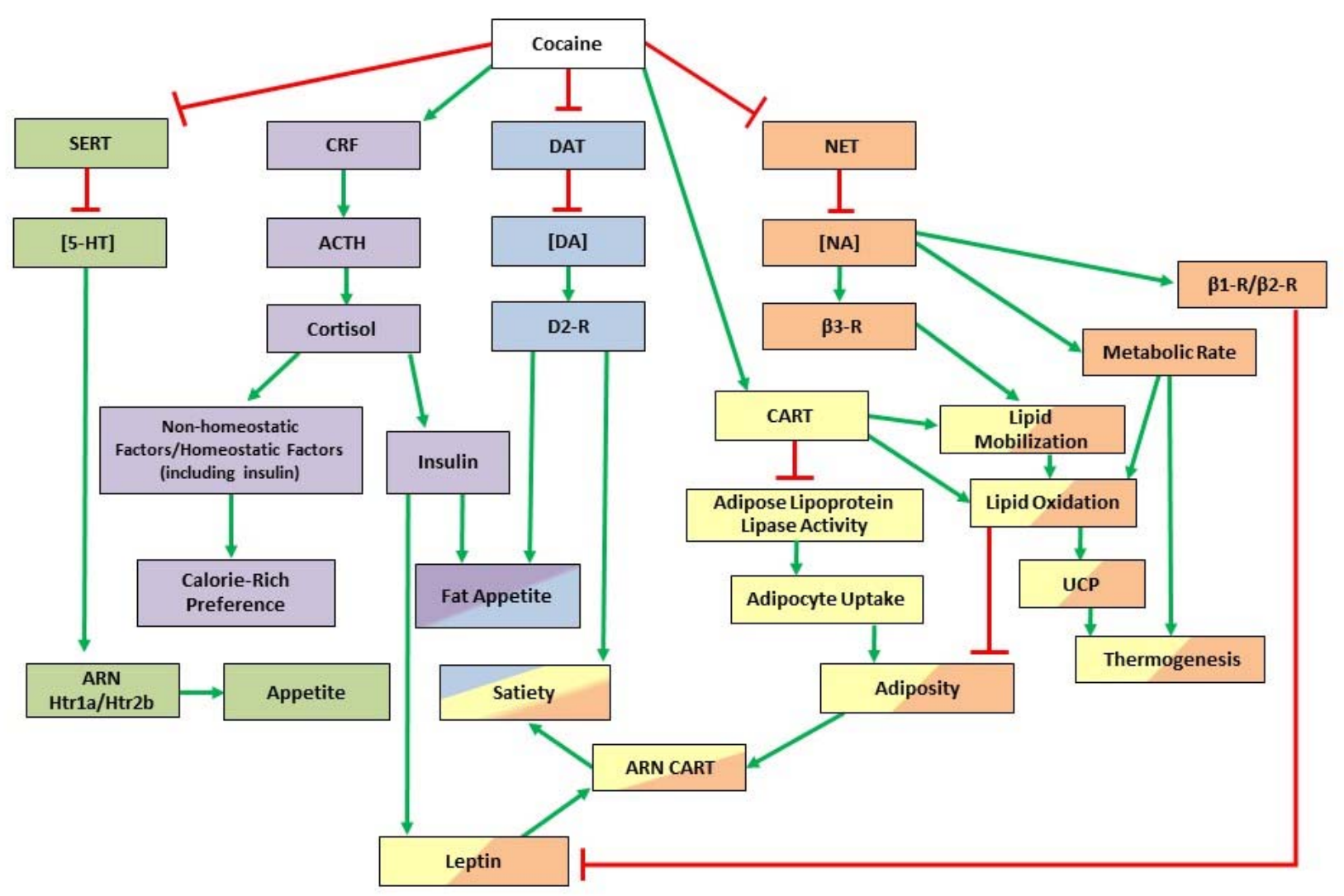

PATHWAYS: Serotonin $\square$ HPA $\square$ Dopamine $\square$ Noradrenaline $\square$ CART $\square$ EFFECTS: Excitatory $\longrightarrow$ Inhibitory $\longrightarrow$

Figure 1: Possible biochemical and physiological effects of cocaine on monoaminergic neurotransmitter systems by inhibiting the reuptake of serotonin (5-HT), dopamine (DA), noradrenaline (NA), as indicated by red bar-headed lines. Blockade of the re-uptake increases the availability of monoamines in the synaptic cleft, as indicated by squared brackets. The excitatory effects of cocaine on the hypothalamic-pituitary-adrenal axis (HPA) and cocaine and amphetamine regulated transcript (CART) pathways are indicated by green arrows. Cocaine promotes serotonergic signaling resulting in an increase in appetite via increased activation of $\mathrm{Htrla} / 2 \mathrm{~b}$ receptors of arcuate nucleus (ARN) neurons. Dopaminergic signaling is enhanced by cocaine, i.e. activation of dopamine D2 receptors (D2-Rs) leads acutely to satiety, but has been suggested to increase fat intake in chronic cocaine users. Cocaine also stimulates CART release, which drives short-lived anorexigenic effects. CART, in turn, initiates increased lipid mobilization and oxidation; this involves a decrease in adipose lipoprotein lipase activity (involved in fat deposition) and uncoupling proteins (UCP) up-regulation. Leptin can also stimulate CART release within selective parts of the brain e.g. ARN. Cocaine modulates the HPA pathway, increasing corticotrophin-releasing factor (CRF) release, resulting in increased adrenocorticotropic hormone $(\mathrm{ACTH})$ release and circulating glucocorticoids. Glucocorticoids alter homeostatic and non-homeostatic factors increasing calorie/fat dietary intake in chronic cocaine users. Cocaine enhances noradrenergic signaling resulting in increased metabolic rate and thermogenesis. Stimulation of beta-3 adrenergic receptors ( $\beta 3$-Rs) and beta- 1 and beta- 2 adrenergic receptors $(\beta 1-\mathrm{R}$ and $\beta 2-\mathrm{R})$, increases thermogenesis and reduces leptin production respectively. 


\section{References}

1. VanBuskirk KA, Potenza MN 2010 The Treatment of Obesity and Its Co-Occurrence With Substance Use Disorders. Journal of Addiction Medicine 4: 1-10

2. Balopole DC, Hansult CD, Dorph D 1979 Effect of Cocaine on Food-Intake in Rats. Psychopharmacology 64: 121-122

3. Bane AJ, McCoy JG, Stump BS, Avery DD 1993 The Effects of Cocaine on Dietary SelfSelection in Female Rats. Physiology \& Behavior 54: 509-513

4. Ersche KD, Stochl J, Woodward JM, Fletcher PC 2013 The skinny on cocaine. Insights into eating behavior and body weight in cocaine-dependent men. Appetite 71: 75-80

5. Castro FG, Newcomb MD, Cadish K 1987 Life-Style Differences Between Young-Adult Cocaine Users and Their Nonuser Peers. Journal of Drug Education 17: 89-111

6. Thanos PK, Cho J, Kim R, Michaelides M, Primeaux S, Bray G, Wang GJ, Volkow ND 2011 Bromocriptine increased operant responding for high fat food but decreased chow intake in both obesity-prone and resistant rats. Behavioural Brain Research 217: 165-170

7. Kenny PJ, Voren G, Johnson PM 2013 Dopamine D2 receptors and striatopallidal transmission in addiction and obesity. Current Opinion in Neurobiology 23: 535-538

8. Ramamoorthy S, Bauman AL, Moore KR, Han H, Yang-Feng T, Chang AS, Ganapathy V, Blakely RD 1993 Antidepressant- and cocaine-sensitive human serotonin transporter: molecular cloning, expression, and chromosomal localization. Proceedings of the National Academy of Sciences 90: 2542-2546

9. Yadav VK, Oury F, Suda N, Liu ZW, Gao XB, Confavreux C, Klemenhagen KC, Tanaka KF, Gingrich JA, Guo XE, Tecott LH, Mann JJ, Hen R, Horvath TL, Karsenty G 2009 A Serotonin-Dependent Mechanism Explains the Leptin Regulation of Bone Mass, Appetite, and Energy Expenditure. Cell 138: 976-989

10. Nieuwenhuizen AG, Rutters F 2008 The hypothalamic-pituitary-adrenal-axis in the regulation of energy balance. Physiology \& Behavior 94: 169-177

11. Marinelli M, Piazza PV 2002 Interaction between glucocorticoid hormones, stress and psychostimulant drugs. European Journal of Neuroscience 16: 387-394

12. Rutters F, Fleur S, Lemmens S, Born J, Martens M, Adam T 2012 The HypothalamicPituitary-Adrenal Axis, Obesity, and Chronic Stress Exposure: Foods and HPA Axis. Current Obesity Reports 1: 199-207

13. Zakrzewska KE, Cusin I, Sainsbury A, RohnerJeanrenaud F, Jeanrenaud B 1997 Glucocorticoids as counterregulatory hormones of leptin - Toward an understanding of leptin resistance. Diabetes 46: 717-719

14. La Fleur SE, Akana SF, Manalo SL, Dallman MF 2004 Interaction between corticosterone and insulin in obesity: Regulation of lard intake and fat stores. Endocrinology 145: 21742185 
15. Schoffelmeer ANM, Drukarch B, De Vries TJ, Hogenboom F, Schetters D, Pattij T 2011 Insulin Modulates Cocaine-Sensitive Monoamine Transporter Function and Impulsive Behavior. Journal of Neuroscience 31: 1284-1291

16. Robertson SD, Matthies HJG, Owens WA, Sathananthan V, Christianson NSB, Kennedy JP, Lindsley CW, Daws LC, Galli A 2010 Insulin Reveals Akt Signaling as a Novel Regulator of Norepinephrine Transporter Trafficking and Norepinephrine Homeostasis. Journal of Neuroscience 30: 11305-11316

17. Niswender KD, Schwartz MW 2003 Insulin and leptin revisited: adiposity signals with overlapping physiological and intracellular signaling capabilities. Frontiers in Neuroendocrinology 24: 1-10

18. Vicentic A, Jones DC 2007 The CART (Cocaine- and Amphetamine-Regulated Transcript) System in Appetite and Drug Addiction. Journal of Pharmacology and Experimental Therapeutics 320: 499-506

19. Rogge G, Jones D, Hubert GW, Lin Y, Kuhar MJ 2008 CART peptides: regulators of body weight, reward and other functions. Nature Reviews Neuroscience 9: 747-758

20. Wortley KE, Chang GQ, Davydova Z, Fried SK, Leibowitz SF 2004 Cocaine- and amphetamine-regulated transcript in the arcuate nucleus stimulates lipid metabolism to control body fat accrual on a high-fat diet. Regulatory Peptides 117: 89-99

21. Rayner DV, Trayhurn P 2001 Regulation of leptin production: sympathetic nervous system interactions. Journal of Molecular Medicine-Jmm 79: 8-20 